\title{
ANÁLISE DE CRITÉRIOS DE QUALIDADE DA MADEIRA DE EUCALIPTO PARA PRODUÇÃO DE CELULOSE KRAFT ${ }^{1}$
}

\author{
Jose Livio Gomide², Humberto Fantuzzi Neto ${ }^{3}$ e Adair José Regazzi ${ }^{4}$
}

\begin{abstract}
Resumo - Setenta e cinco amostras de madeiras de clones de Eucalyptus, obtidas de diferentes regiões geográficas do Brasil, foram utilizadas para este estudo. Análises estatísticas demonstraram que a carga de álcali ativo para polpação, o teor de lignina e o teor de extrativos apresentaram efeitos significativos no rendimento da polpação kraft. A densidade básica da madeira não afetou significativamente o rendimento da polpação, mas sim o consumo de madeira para produção de celulose $\left(\mathrm{m}^{3}\right.$ madeira/t celulose). Densidade básica, teor de lignina e teor de extrativos foram recomendados como critérios de qualidade da madeira a serem determinados para seleção de clones antes da determinação das características de polpação.
\end{abstract}

Palavras-chave: Eucalyptus, Qualidade da madeira e Rendimento kraft.

\section{ANALYSIS OF WOOD QUALITY CRITERIA OF EUCALYPTUS WOOD FOR KRAFT PULP PRODUCTION}

\begin{abstract}
Seventy five Eucalyptus wood clones obtained from different geographic regions in Brazil were used for this study. Based on statistical analysis it was demonstrated that an active alkali charge and also wood lignin and extractive contents presented a significant effect on pulping yield. Wood specific gravity did not present a significant effect on pulping yield but affected wood consumption for pulp production ( $\mathrm{m}^{3}$ wood/ ton pulp). Wood specific gravity, lignin content and extractive content were recommended as criteria to establish wood quality prior to pulping characteristics determination.
\end{abstract}

Keywords: Eucalyptus, Wood quality and Pulping yield.

\section{INTRODUÇÃO}

O Brasil ocupa, atualmente, a quarta posição entre os maiores produtores mundiais de celulose. Segundo dados da Bracelpa, referentes a 2008, a produção nacional de celulose, incluindo as pastas de alto rendimento, atingiu nesse ano a cifra de 12,7 milhões de toneladas. Dessa produção, mais de 96\% foi de polpa celulósica química. A produção nacional de polpa química foi alcançada predominantemente com madeiras de eucalipto que representaram mais de $86 \%$ da produção total, tendo as madeiras de Pinus sido responsáveis pelos restantes 14\%. O formidável crescimento da indústria nacional de celulose deverá continuar nos próximos anos e existem previsões de que em 2012 a produção nacional de celulose deverá ultrapassar 14 milhões de toneladas, posicionando o Brasil como o quarto maior produtor mundial de celulose.
O Brasil apresentou, nas últimas décadas, uma invejável evolução na produtividade das florestas de Eucalyptus, passando de $15 \mathrm{~m}^{3} / \mathrm{ha}$ /ano na década de 1970 para, atualmente, uma média nacional de cerca de $45 \mathrm{~m}^{3} / \mathrm{ha} / \mathrm{ano}$. Estudo recente de caracterização dos melhores clones de Eucalyptus cultivados no Brasil (GOMIDE et al., 2005), envolvendo as principais indústrias nacionais de celulose, demonstrou que esses plantios florestais no Brasil apresentam os mais elevados níveis mundiais de incremento médio anual (IMA). Os clones de Eucalyptus analisados neste estudo demonstram elevada produtividade, tendo $20 \%$ dos clones atingido IMA igual ou superior a $50 \mathrm{~m}^{3} / \mathrm{ha} / \mathrm{ano}$, e $70 \%$ dos clones apresentaram incrementos acima de $40 \mathrm{~m}^{3} / \mathrm{ha} /$ ano.

\footnotetext{
${ }^{1}$ Recebido em 25.11.2008 e aceito para publicação em 14.10.2009.

${ }^{2}$ Departamento de Engenharia Florestal, Universidade Federal de Viçosa, UFV, Brasil. E-mail: <jlgomide@ufv.br>.

${ }^{3}$ Universidade Federal do Espírito Santo, UFES, Brasil.

${ }^{4}$ Departamento de Informática, Universidade Federal de Viçosa, UFV, Brasil. E-mail: <adairreg@ufv.br>.
} 
Esses elevados patamares de produtividade foram alcançados principalmente pela utilização de técnicas de clonagem. Os estudos de clonagem de Eucalyptus sp. para estabelecimento de florestas com a qualidade requerida pelas empresas requerem análises e testes de milhares de clones potenciais. A seleção de clones inicia-se com os aspectos silviculturais e, depois de uma seleção preliminar, pelas características silviculturais é indispensável a realização de análises tecnológicas para estabelecimento da qualidade da madeira de, ainda, centenas de clones remanescentes. Apenas depois desses estudos silviculturais e tecnológicos é que são selecionados os clones para multiplicação e formação de florestas homogêneas que irão apresentar, após um período de rotação de cinco a sete anos, alta produtividade florestal e, também, alta qualidade tecnológica para produção de polpa celulósica.

Os estudos de caracterização de qualidade da madeira para produção de polpa celulósica envolvem a constituição química da madeira, suas características estruturais anatômicas e, também de grande importância, as características tecnológicas da transformação da madeira em polpa celulósica. Estudos completos e exaustivos de todas as características tecnológicas dos clones em fase de seleção são extremamente dispendiosos e requerem tempos excessivamente longos, inviabilizando a realização completa desses estudos. Madeira de um único clone poderia representar custo de alguns milhares de dólares para ser completamente caracterizada nas suas propriedades físicas, químicas e tecnológicas para produção de polpa celulósica, conforme demonstrado por Downes et al. (1997). Entretanto, apesar de dispendiosa, a caracterização da madeira é de fundamental importância e terá que, inevitavelmente, ser realizada. Normalmente, a solução desses problemas consiste na realização de apenas algumas das análises, consideradas indispensáveis, capazes de fornecerem os subsídios necessários para uma seleção tecnicamente consciente. Normalmente, numa primeira etapa de seleção de clones por critérios tecnológicos de qualidade de madeira são recomendados os estudos de densidade básica, de constituição química fundamental da madeira e, inevitavelmente, de transformação da madeira em polpa celulósica.

No Brasil, normalmente são determinados a densidade básica da madeira, o teor de lignina Klason, insolúvel e solúvel, e o teor de extrativos solúveis em uma mistura álcool e tolueno ou, mais recentemente, em acetona. Essas análises poderiam ser consideradas como caracterização básica da madeira, compreendendo um mínimo necessário de informações para uma caracterização preliminar de qualidade da madeira. Estudos de polpação kraft da madeira são, certamente, de grande importância, mas representam substancial acréscimo nos custos de caracterização de qualidade da madeira. Nos estudos de polpação da madeira, como mencionado por Gomide e Colodette (2007), o rendimento do processo poderia ser considerado como o principal parâmetro de qualidade da madeira, uma vez que está diretamente relacionado com as estruturas anatômica e química da madeira, a demanda de carga alcalina, a produção do digestor e a carga de sólidos para a caldeira de recuperação.

O objetivo deste estudo foi verificar se as análises de caracterização básica da madeira podem ser correlacionadas com o rendimento de polpação da madeira. Se confirmadas correlações significativas, poderia ser indicada apenas a realização dessas análises básicas, para a totalidade dos clones, na etapa inicial da seleção de clones num programa de melhoramento florestal. Os clones selecionados nessa primeira etapa é que seriam indicados para caracterização posterior mais detalhada de polpação kraft.

\section{MATERIAL E MÉTODOS}

Para a realização deste estudo, foram utilizadas 75 amostras de madeiras de Eucalyptus, em idade comercial de corte, fornecidas por empresas nacionais de celulose, localizadas em diferentes regiões do Brasil, mas sem especificação por local de coleta. Três árvores de cada clone de Eucalyptus sp., apresentando DAP e alturas correspondentes às médias do povoamento, foram seccionadas em cinco toretes de $50 \mathrm{~cm}$ de comprimento, retirados na base e a 25, 50, 75 e 100\% da altura comercial. A altura comercial da árvore foi considerada todo o tronco até a altura correspondente ao diâmetro de $6 \mathrm{~cm}$. Os toretes foram transformados em cavacos por um picador laboratorial de disco com três facas, dotado de duas peneiras classificadoras com aberturas de 39 x 39 mm e 6 x 6 mm. Os cavacos foram reclassificados em peneira com abertura de 16 x 16 mm, sendo realizada, também, a classificação manual para eliminar pedaços de casca, cavacos com defeitos de corte e madeiras com nós. Depois de selecionados, os cavacos foram secos ao ar, e, para alcançar completa homogeneização, utilizou-se um misturador esférico mecânico com capacidade para 260 L, onde os 
cavacos foram intensamente misturados. Finalmente, os cavacos foram armazenados em sacos de polietileno, para uniformização e conservação do teor de umidade.

Uma amostra dos cavacos de cada clone foi transformada em serragem, utilizando-se moinho Wiley, e a serragem foi classificada em peneiras de 40 e 60 mesh. As análises químicas das serragens foram realizadas após acondicionamento em sala climatizada a $25^{\circ} \mathrm{C}$ e $50 \%$ de umidade relativa. As metodologias utilizadas para determinação da densidade básica e para as análises químicas foram as estabelecidas nas normas ABNTe TAPPI ou conforme especificado a seguir: densidade básica, conforme norma ABNT(NBR 11941); solubilidade em álcool/tolueno, norma Tappi T264 om-82; lignina Klason insolúvel em ácido, conforme Gomidee Demuner(1986); e lignina Klason solúvel em ácido, conforme Goldschmid (1971).

Os cozimentos kraft foram realizados em digestor rotativo, com quatro reatores individuais de $2 \mathrm{~L}$ cada um, aquecido eletricamente e dotado de termômetro e manômetro, possibilitando a realização, numa única batelada, de quatro cozimentos simultaneamente. O tempo e temperatura de cozimento foram monitorados por meio de controlador eletrônico acoplado a um computador. O sensor de temperatura foi um PT100, em contato direto com os cavacos e o licor de cozimento. Em cada reator foi utilizada carga diferente de álcali ativo $(17,19,21$ e 23\%), como $\mathrm{NaOH}$, para estabelecer a curva de deslignificação da madeira. Foram realizados, simultaneamente, quatro cozimentos em cada batelada. Essas cargas de álcali foram selecionadas após a realização de cozimentos exploratórios, objetivando obtenção de número kappa próximo a 18 na parte mediana da curva de deslignificação. Os outros parâmetros utilizados para a polpação kraft foram os seguintes, mantidos constantes para todos os cozimentos: cavacos $=250$ g a.s., sulfidez $=25 \%$, relação licor $/$ madeira $=4 / 1$, temperatura máxima $=170^{\circ} \mathrm{C}$, tempo até a temperatura máxima $=90 \mathrm{~min}$, tempo à temperatura máxima $=50 \mathrm{~min}$. Na Figura 1 é apresentado o gráfico dos perfis de temperatura e de fator $\mathrm{H}$, determinados eletronicamente para cada cozimentos kraft. Foi desenvolvida uma análise de regressão de cada conjunto de quatro cozimentos, realizados simultaneamente para cada clone, estabelecendo-se equações para correlacionar número kappa, rendimento depurado e viscosidade em função do álcali ativo aplicado. Pelo uso das equações, foi determinado o álcali ativo necessário para obter número kappa 18,0, bem como o rendimento do processo e a viscosidade da polpa correspondentes ao número kappa 18,0. Uma descrição detalhada da metodologia utilizada para os cozimentos está apresentada em publicação de Gomide et al. (2004).
As seguintes características foram avaliadas: densidade básica da madeira $\left(\mathrm{kg} / \mathrm{m}^{3}\right)$, teor de extrativos (\%), teor de lignina (\%), álcali ativo para obtenção de kappa 18,0 (\%), viscosidade (cP), rendimento (\%) e consumo específico de madeira ( $\mathrm{m}^{3} / \mathrm{t}$ celulose). Os dados foram analisados estatisticamente, por meio de análises de correlação e de regressão. Como todas as características avaliadas eram variáveis aleatórias, foi feito estudo de correlação para avaliar o grau de associação entre essas variáveis, em que se admitiu um nível de significância de $5 \%$ de probabilidade pelo teste t de Student. Numa segunda etapa, foram ajustadas equações de regressão para rendimento, tendo como variáveis explicativas a densidade básica da madeira, o teor de extrativos, o teor de lignina e álcali ativo. Também, foram ajustadas equações de regressão para consumo específico, tendo como variáveis explicativas a densidade básica da madeira, o teor de extrativos e o teor de lignina. A escolha da melhor equação ajustada foi feita utizando-se o procedimento de seleção por etapas ("stepwise regression procedure”) e admitindo um nível de significância de até $10 \%$ de probabilidade. Todas as análises estatísticas foram realizadas utilizando-se o programa SAS (“Statistical Analysis System”).

\section{PERFIL DE TEMPERATURA E FATOR H}

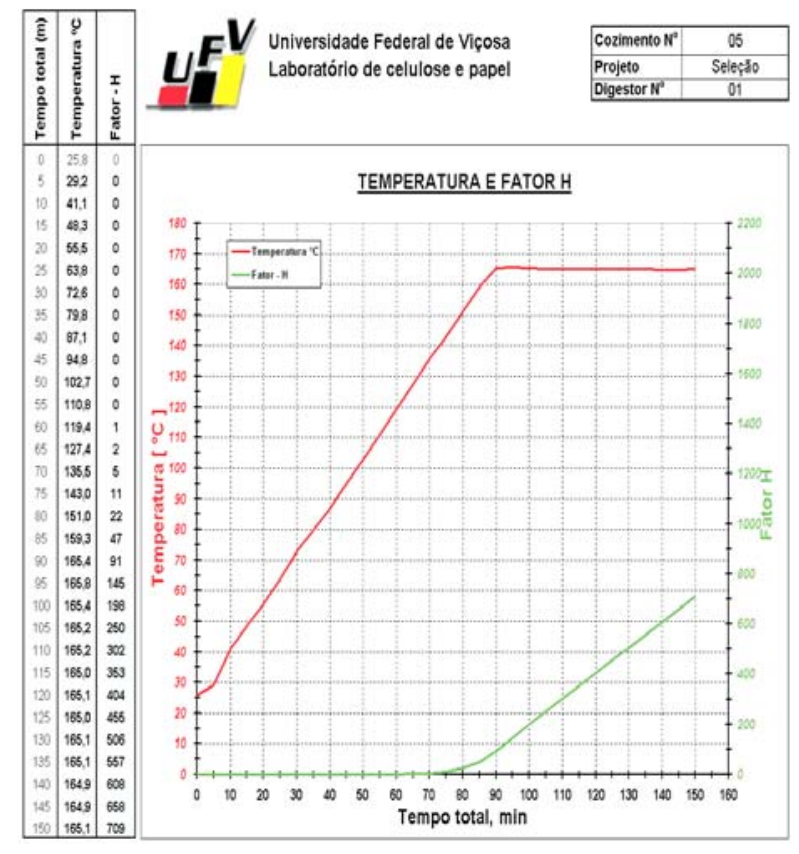

Figura 1 - Perfis de temperatura e de fator $\mathrm{H}$ do cozimento kraft. Figure 1-Temperature and $H$ factor profiles for kraft cooking.

R. Árvore, Viçosa-MG, v.34, n.2, p.339-344, 2010 


\section{RESULTADOS E DISCUSSÃO}

Na Tabela 1 são apresentados os resultados das análises tecnológicas das madeiras dos 75 clones analisados, incluindo os valores de média, máximo, mínimo e desvio-padrão para densidade básica, teor de lignina Klason, teor de extrativos em álcool/tolueno, demanda de álcali ativo para obtenção de kappa 18, rendimento no kappa 18, viscosidade da polpa com kappa 18 e consumo específico de madeira.

Como mostrado na Tabela 1 , a variabilidade das características tecnológicas dos clones analisados foi bastante ampla, bastando citar, como exemplo, que o rendimento variou desde $44,6 \%$ até $53,1 \%$, demonstrando a grande importância da qualidade da madeira num programa de melhoramento florestal. A demanda de álcali para alcançar kappa 18 também variou fortemente entre os clones, desde 16,9\% até 23,7\%. Variações na qualidade da madeira podem afetar significativamente o processo fabril industrial, desde a produção do digestor e o desempenho da caldeira de recuperação até a qualidade da polpa celulósica.

Para compreender melhor o efeito das características de qualidade da madeira, representadas neste estudo pela densidade básica, teor de lignina e teor de extrativos, no processo de produção de celulose, foram realizadas várias análises estatísticas. As estimativas dos coeficientes de correlação linear simples entre todas as variáveis de qualidade da madeira analisadas estão apresentadas na Tabela 2.

Na Tabela 2 pode ser observado que a maioria das correlações foi estatisticamente significativa pelo teste t. Algumas estimativas foram superiores a 0,50 (em valor absoluto), como as correlações entre álcali e extrativos $(0,51)$, lignina e consumo específico $(0,61)$, rendimento e viscosidade $(0,70)$, extrativos e rendimento $(-0,72)$, álcali e viscosidade $(-0,73)$, densidade e consumo específico $(-0,76)$ e, finalmente, álcali e rendimento $(-0,86)$. A correlação entre a carga de álcali e o rendimento de -0,86 demonstra que aumentos do álcali proporcionam significativa queda no rendimento do processo. A correlação de -0,76 entre a densidade e o consumo específico demonstra a importância da densidade no processo de produção de celulose, uma vez que madeiras mais densas proporcionam menor consumo de madeira ( $\mathrm{m}^{3} \mathrm{t}$ celulose), o que favorece a produção de celulose no digestor e a manutenção de volume da pilha de cavacos nas fábricas. Uma característica da madeira que se mostrou bastante influente no rendimento foi o teor de extrativos, com correlação de $-0,72(p<0,05)$, o que é compreensível,

Tabela 1 -Características tecnológicas dos clones de Eucalyptus.

Table 1 - Technological characteristics of Eucalyptus wood.

\begin{tabular}{|c|c|c|c|c|}
\hline Características & Média & Mínimo & Máximo & Desvio-padrão \\
\hline Densidade básica, kg/m³ & 493 & 429 & 555 & 26,48 \\
\hline Teor extrativos, \% & 3,08 & 1,24 & 7,29 & 1,27 \\
\hline Teor lignina (total), \% & 27,3 & 23,3 & 30,9 & 1,77 \\
\hline Álcali ativo, \% & 20,1 & 16,9 & 23,7 & 1,37 \\
\hline Rendimento, \% & 49,5 & 44,6 & 53,1 & 1,99 \\
\hline Viscosidade, cP & 41,9 & 27,2 & 61,0 & 7,23 \\
\hline Consumo específico (m³/t cel) & 4,11 & 3,69 & 4,71 & 0,26 \\
\hline
\end{tabular}

Tabela 2 - Estimativas dos coeficientes de correlação linear simples entre as variáveis. Table 2 - Single linear correlation coefficients.

\begin{tabular}{|c|c|c|c|c|c|c|c|}
\hline & Densidade & Extrativos & Lignina & Álcali ativo & Rendimento & Viscosidade & $\begin{array}{c}\text { Cons. } \\
\text { Específico }\end{array}$ \\
\hline$\overline{\text { Densidade, } \mathrm{kg} / \mathrm{m}^{3}}$ & 1,00 & $0,24^{*}$ & $-0,36^{*}$ & $0,30^{*}$ & $-0,17^{\mathrm{ns}}$ & $-0,11^{\mathrm{ns}}$ & $-0,76^{*}$ \\
\hline Extrativos, \% & $0,24^{*}$ & 1,00 & $0,09^{\mathrm{ns}}$ & $0,51^{*}$ & $-0,72^{*}$ & $-0,38^{*}$ & $0,27^{*}$ \\
\hline Lignina, \% & $-0,36^{*}$ & $0,09^{\text {ns }}$ & 1,00 & $0,33^{*}$ & $-0,45^{*}$ & $-0,19^{\text {ns }}$ & $0,61^{*}$ \\
\hline Álcali ativo, \% & $0,30^{*}$ & $0,51^{*}$ & $0,33^{*}$ & 1,00 & $-0,86^{*}$ & $-0,73^{*}$ & $0,30^{*}$ \\
\hline Rendimento, \% & $-0,17^{\mathrm{ns}}$ & $-0,72^{*}$ & $-0,45^{*}$ & $-0,86^{*}$ & 1,00 & $0,70^{*}$ & $-0,51^{*}$ \\
\hline Viscosidade, \% & $-0,11^{\mathrm{ns}}$ & $-0,38^{*}$ & $-0,19^{\mathrm{ns}}$ & $-0,73^{*}$ & $0,70^{*}$ & 1,00 & $-0,35^{*}$ \\
\hline Cons. Específico, $\mathrm{m}^{3} / \mathrm{t}$ & $-0,76^{*}$ & $0,27^{*}$ & $0,61^{*}$ & $0,30^{*}$ & $-0,51^{*}$ & $-0,35^{*}$ & 1,00 \\
\hline
\end{tabular}

* - Significativo a 5\% de probabilidade, pelo teste $\mathrm{t}$.

ns - Não significativo a 5\% de probabilidade, pelo teste t.

R. Árvore, Viçosa-MG, v.34, n.2, p.339-344, 2010 
uma vez que ocorre intensa remoção desses constituintes durante a polpação. O teor de lignina afetou negativamente o rendimento, com uma correlação $-0,45(p<0,05)$. Deve ser considerado que os teores de lignina nas madeiras (23,3\% a 30,9\%) foram muito superiores que os extrativos (1,24\% a 7,29\%). Ambas as correlações foram significativas, evidenciando a importância dos extrativos e da lignina como critérios de qualidade da madeira de Eucalyptus para produção de celulose.

A viscosidade correlacionou-se positivamente com o rendimento $(0,70)$, uma vez que rendimentos mais altos, obtidos com menores álcalis, estão relacionados com menor degradação dos carboidratos e, consequentemente, com viscosidade mais elevada. A densidade básica da madeira apresentou correlação positiva e significativa com a carga de álcali $(0,30)$. Essa correlação é um indicativo de que madeiras de densidades mais altas podem requerer álcali mais elevado no cozimento. A correlação da densidade com o rendimento $(-0,17)$ não foi significativa $(p>0,05)$.

A densidade básica da madeira é, provavelmente, a característica de qualidade utilizada com mais frequência nos programas de seleção de clones para melhoramento florestal, o que é justificável pela sua alta correlação com o consumo específico $\left(\mathrm{m}^{3}\right.$ de madeira por tonelada de celulose). Entretanto, nos últimos anos tem sido questionada a importância da densidade da madeira no rendimento do digestor. Seria, portanto, interessante responder neste estudo ao seguinte questionamento: “A densidade básica da madeira de Eucalyptus tem ou não efeito no rendimento?”

Para responder a esse questionamento e considerando a grande importância do rendimento de polpação para estabelecimento de critérios de qualidade da madeira, foram ajustadas várias equações de regressão para rendimento em função da densidade básica da madeira, do teor de extrativos, do teor de lignina e do álcali ativo aplicado, como mostrado na Tabela 3. Pela técnica de
“Seleção por Etapas”, a equação selecionada foi a número 2, que apresentou o coeficiente de determinação mais elevado $\left(\mathrm{R}^{2}=89,2 \%\right)$, sendo a variável álcali a primeira a entrar no modelo, com $\mathrm{R}^{2}$ parcial de $74,4 \%$, seguida, respectivamente, por extrativos $\left(R^{2}\right.$ parcial $\left.=10,3 \%\right)$ e lignina $\left(\mathrm{R}^{2}\right.$ parcial $\left.=4,5 \%\right)$.

Na equação 1 da Tabela 3 é apresentado o modelo completo, onde todas as variáveis analisadas foram consideradas. Analisando as equações 1 e 2, verificase que os valores de $\mathrm{R}^{2}$ ajustados foram idênticos. Entretanto, na equação 1 o efeito da densidade básica foi não significativo ( $p>0,05$ ), indicando que a densidade básica pode ser eliminada do modelo, uma vez que seu efeito no rendimento do processo seria desprezível. Assim, com as variáveis álcali, extrativos e lignina presentes no modelo, a densidade não contribuiria para explicar a variação observada na variável resposta que é o rendimento. Na equação 5, apesar de os coeficientes de regressão serem significativos e como a variável álcali não está presente, o $\mathrm{R}^{2}$ caiu fortemente, evidenciando a importância dessa variável no processo.

Como o rendimento afeta o consumo específico de madeira, foram realizadas análises estatísticas por meio de equações de regressão ajustadas para consumo específico em função da densidade básica, do teor de lignina e do teor de extrativos (Tabela 4). A equação 4, estabelecida sem a densidade básica, apresentou o menor $\mathrm{R}^{2}$ ajustado, indicando que esse parâmetro é de fundamental importância na predição do consumo específico. Entretanto, os teores de lignina e de extrativos devem ser também considerados, como demonstrado pela equação 1 , em que o $\mathrm{R}^{2}$ ajustado foi o mais elevado. Pela técnica de “Seleção por Etapas”, a equação selecionada foi a número 1 , com coeficiente de determinação $R^{2}=87,0 \%$, sendo a variável densidade a primeira a entrar no modelo, com $\mathrm{R}^{2}$ parcial de $58,0 \%$, seguida, respectivamente, por extrativos $\left(R^{2}\right.$ parcial $\left.=21,5 \%\right)$ e lignina $\left(R^{2}\right.$ parcial $\left.=7,5 \%\right)$.

Tabela 3 - Equações de regressão ajustadas para o rendimento depurado.

Table 3 - Regression equations adjusted for screened yields.

\begin{tabular}{llcc}
\hline & Equações ajustadas & $\mathrm{R}^{2}$ & $\mathrm{R}_{\text {ajust. }}^{2}(\%)$ \\
\hline 1 & $\mathrm{RD} \%=74,01+0,00264^{\mathrm{ns}} \mathrm{Db}-0,23135^{* *} \mathrm{Lig}-0,62373^{* *}$ Ext $-0,87189^{* *} \mathrm{AA}$ & 89,2 & 88,6 \\
2 & $\mathrm{RD} \%=75,40-0,25141^{* *} \mathrm{Lig}-0,62020^{* *}$ Ext $-0,84972^{* *} \mathrm{AA}$ & 89,2 & 88,7 \\
3 & $\mathrm{RD} \%=66,83+0,01039^{* *} \mathrm{Db}-0,60897^{* *}$ Ext $-1,02158^{* *} \mathrm{AA}$ & 86,4 & 85,8 \\
4 & $\mathrm{RD} \%=78,35+0,00056^{\mathrm{ns}} \mathrm{Db}-0,20876^{* *} \mathrm{Lig}-1,16314^{* *} \mathrm{AA}$ & 77,6 & 76,7 \\
5 & $\mathrm{RD} \%=73,48-0,01372^{*} \mathrm{Db}-0,51729^{* *} \mathrm{Lig}-0,98724^{* *}$ Ext & 69,0 & 67,7 \\
\hline
\end{tabular}

Rendimento depurado (RD\%), densidade básica (Db), lignina (Lig), extrativo (Ext) e álcali ativo (AA).

$* \mathrm{e}^{* *}$ - Significativos a 5\% e $1 \%$ de probabilidade, respectivamente, pelo teste $\mathrm{t}$.

ns - Não significativo a $5 \%$ de probabilidade, pelo teste t. 
Tabela 4-Equações de regressão ajustadas para consumo específico.

Table 4 - Regression equations for wood specific consumption.

\begin{tabular}{|c|c|c|c|}
\hline & Equações ajustadas & $\mathrm{R}^{2}(\%)$ & $\mathrm{R}_{\text {ajust. }}^{2}(\%)$ \\
\hline 1 & $\mathrm{CE}=6,27-0,00733{ }^{* *} \mathrm{Db}+0,08544^{* *} \mathrm{Ext}+0,04345^{* *} \mathrm{Lig}$ & 87,0 & 86,6 \\
\hline 2 & $\mathrm{CE}=8,01-0,00851{ }^{* *} \mathrm{Db}+0,09680^{* *} \mathrm{Ext}$ & 79,5 & 78,9 \\
\hline 3 & $\mathrm{CE}=5,57-0,00605^{* *} \mathrm{Db}+0,05586^{* *} \mathrm{Lig}$ & 70,9 & 70,1 \\
\hline 4 & $\mathrm{CE}=1,64+0,04335^{*} \mathrm{Ext}+0,08561{ }^{* *} \mathrm{Lig}$ & 41,7 & 40,1 \\
\hline
\end{tabular}

Consumo específico (CE), densidade básica (Db), extrativo (Ext) e lignina (Lig).

* e e* $^{*}$ - Significativos a $5 \%$ e $1 \%$ de probabilidade, respectivamente, pelo teste $t$.

\section{CONCLUSÕES}

A análise global dos resultados e as respectivas análises estatísticas demonstraram que o teor de lignina e o teor de extrativos são importantes características de qualidade da madeira, afetando significativamente o rendimento do processo de polpação. A densidade básica da madeira demonstrou não estar correlacionada significativamente com o rendimento da polpação, mas apresentou correlação significativa com o consumo da madeira para produção de celulose. Assim, num programa de melhoramento florestal seria indicado determinar, inicialmente, essas características de qualidade da madeira (densidade básica, teor de lignina e teor de extrativos), previamente aos estudos de polpação que apresentam custo bem mais elevado.

\section{REFERÊNCIAS}

\section{ASSOCIAÇÃO BRASILEIRA DE NORMAS}

TÉCNICAS - ABNT-. Rio de Janeiro: 2002.

DOWNES, G. M. et al. Sampling plantation Eucalyptus for wood and fiber properties. Melbourne:CSIRO Publishing, 1997. 132p.
GOLDSCHMID. Ultraviolet spectra. In: SARKANEN, K. V.; LUDWIG, C. H. (Eds.) Lignins. New York: Wiley-Interscience, 1971. p.241-266.

GOMIDE, J. L.; COLODETTE, J. L. Qualidade da madeira. In: BOREM, A. (Ed.) Biotecnologia florestal. Viçosa, MG: Universidade Federal de Viçosa, 2007. p.25-54.

GOMIDE, J. L.; DEMUNER, B. J. Determinação do teor de lignina em material lenhoso: Método Klason modificado. O Papel, v.47, n.8, p.36-38, 1986.

GOMIDE, J. L. et al. Caracterização tecnológica para produção de celulose da nova geração de clones de Eucalyptus do Brasil. Revista Árvore, v.29, n.1, p.129-137, 2005.

GOMIDE, J. L.; FANTUZZI NETO, H.; LEITE, H. G. Estratégia de análise da qualidade da madeira de Eucalyptus sp para produção de celulose kraft. Revista Árvore, v.28, n.3, p.443-4507, 2004.

TECHNICAL ASSOCIATION OF THE PULP AND PAPER INDUSTRY - TAPPI. Tappi test

methods: 1992-1993. Atlanta: 1992. 